\title{
Estudio De Percepción De Emprendedores Universitarios Sobre EI Impacto De Un Programa Escolar De Emprendimiento En Sus Empresas, En Tehuipango, Veracruz
}

\section{Francisco Javier Mejía-Ochoa \\ Rogelio Limón-Rivera}

Docentes Investigadores del Instituto Tecnológico Superior de Zongolica y Estudiantes de Doctorado en Ciencias de la Gestión Estratégica en Colegio

Interdisciplinario de Especialización A. C. (CIES), Mexico

\section{Juan Carlos Rojas-Martínez}

Docente Investigador del Instituto Tecnológico Superior de Zongolica,

Mexico

\section{Elyana Cristina Peláez-Muñoz \\ Silvia Hernández-Velásquez. \\ Evaristo Fierro-Domínguez.}

Estudiantes de Doctorado en Ciencias de la Gestión Estratégica en Colegio Interdisciplinario de Especialización A. C. (CIES), Mexico

\section{Dulce María Panzo-Macuixtle}

Lorenzo Chipahua-Chipahua

Estudiantes del Instituto Tecnológico Superior de Zongolica, CeDE

Tehuipango, Veracruz, Mexico

\section{Manuel González-Pérez.}

Doctor Investigador SNI1. UPAEP México

Doi: 10.19044/esj.2017.v13n34p48 URL:http://dx.doi.org/10.19044/esj.2017.v13n34p48

\begin{abstract}
Entrepreneurship has impacts on countries, generating jobs and socioeconomic development. In Mexico, from the 1980s, Higher Education Institutions develop entrepreneurship and self-employment promotion programs, to promote entrepreneurial philosophy among their students. In the State of Veracruz, since 2015 the Higher Technological Institute of Zongolica has developed the Social Entrepreneur and Self-Employment (SES) program. The objective of this research is to conduct a study of the perception of university entrepreneurs on the impact of the SES school program on their companies, in the municipality of Tehuipango, Veracruz.
\end{abstract}


For which a questionnaire was designed, applied and validated to a population of 32 graduates of the entrepreneur program. It is concluded that the respondents agree with being entrepreneurs, consider having influenced socioeconomically in their municipality and perceive that the social entrepreneur and self-employment program influenced their level of entrepreneurship. Finally, it was identified the need to create a program of accompaniment to companies, as support so that they continue operating in the market.

Keywords: Perception, University Entrepreneurs, Entrepreneurship Program, Companies

\section{Resumen}

El emprendimiento tiene impactos en los países, generando empleos y desarrollo socioeconómico. En México, a partir de la década de 1980, las Instituciones de Educación Superior desarrollan programas de emprendimiento y fomento al autoempleo, con el fin de promover la filosofía emprendedora en sus estudiantes. En el Estado de Veracruz, el Instituto Tecnológico Superior de Zongolica ha desarrollado desde el año 2015 el programa Emprendedor Social y Autoempleo (ESA). El objetivo de la presente investigación es realizar un estudio de percepción de emprendedores universitarios sobre el impacto del programa escolar ESA en sus empresas, en el municipio de Tehuipango, Veracruz. Para lo cual se diseñó, aplicó y validó un cuestionario a una población de 32 egresados del programa emprendedor. Se concluye que los encuestados están de acuerdo con ser emprendedores, consideran haber influido socioeconómicamente en su municipio y perciben que el programa emprendedor social y autoempleo realmente influyó en su nivel de emprendimiento. Finalmente, se identificó la necesidad de crear un programa de acompañamiento a empresas, como apoyo para que continúen funcionando en el mercado.

Palabras-clave: Percepción, Emprendedores Universitarios, Programa de Emprendimiento, Empresas

\section{Introducción}

El emprendimiento tiene impacto en los países generando empleos y desarrollo socioeconómico. Para poder estar a la par de las nuevas políticas nacionales y globales que conforman un ecosistema emprendedor, es necesario reflexionar sobre los aspectos inmersos en el proceso de cambio en toda institución. Los cambios institucionales involucran aspectos organizacionales, sociales y culturales. Estos aspectos, a su vez, se estudian 
con base en tres ejes: creatividad, innovación y emprendimiento (Maldonado et al., 2017; Montiel y Lucio, 2017).

En este sentido, las universidades tienen la tarea de formar emprendedores; la formación la realizan al ofrecer servicios de manera independiente, crear empresas e innovar las existentes. Sin embargo, enfrentan desafíos relacionados con el proceso de aprendizaje. Los tres grandes desafíos son: 1) cambio de paradigmas (relaciones nuevas entre personas y el mundo); 2) el entorno económico que requiere de iniciativa empresarial y; 3) una demanda creciente entre empleadores de profesionales. (Gonzalez et al., 2017a; Castillo et al., 2016)

Los cambios de paradigmas en los universitarios se han estudiado bajo diversos enfoques. El primero, consiste en la intención de los estudiantes de crear una empresa o trabajar por cuenta propia. Para abordar la intención de los alumnos de emprender, las universidades deben evaluar a los estudiantes considerando el género, la familia, la experiencia laboral, la educación en autoempleo, el apoyo social, la percepción de barreras y los valores individualistas y colectivistas. Por otro lado, las actitudes emprendedoras son: asunción al riesgo, control interno percibido, necesidad de logro, autoestima e innovación en estudiantes desde su ingreso a la universidad hasta tres años académicos. En este sentido, la intención y actitud de emprender disminuye conforme los estudiantes avanzan en los grados escolares. El segundo enfoque, se refiere a la edad de los emprendedores. Los alumnos de recién incorporación a la universidad son más emprendedores en comparación con los compañeros más veteranos. El tercer enfoque, consiste en la adecuación de los programas de estudio de las universidades. La adecuación es importante para desarrollar la filosofía emprendedora en los estudiantes (Moriano et al., 2006; Krauss, 2011; Olmos y Castillo, 2007; Castillo et al., 2016; Hernández et al., 2017a).

El entorno económico es un factor que incide en la intención de emprender. Los elementos del entorno económico pueden ser sociodemográficos (edad, estado civil, semestre, conocimiento del Programa Emprendedor), psicológicos (autoestima, innovación y propensión al riesgo) y socioeconómicos (tendencia a la inversión), y varían de acuerdo con el contexto (Millán et al., 2017).

La demanda de trabajo para profesionales, por su lado, provoca que el emprendimiento sea una alternativa para desarrollar nuevas empresas y ampliar las oportunidades laborales. El desarrollo de emprendedores impacta en el índice de desempleo en egresados universitarios, así como en la perspectiva y concepción de emprendimiento formal e informal. Lo anterior se impulsa con los programas de incubación de empresas de las universidades. Por ejemplo, la Universidad Autónoma del Estado de México, analizó su estructura institucional con el gobierno y empresas, con el fin de 
identificar sus programas de emprendimiento e incentivos a la incubación, programas de investigación y formación de recursos humanos. Asimismo, las ingenierías en Cómputo y la Licenciatura en Administración, son las que poseen mejores competencias para la auto-empleabilidad. Esto incide en mayor porcentaje de desarrollo de los estudiantes en su profesión. Sin embargo, algunos estudios muestran que los factores psicosociales inciden en que los estudiantes, en general, tengan más intención a emplearse, y menos a desarrollar su carrera a través del autoempleo (Moriano et al., 2006; Jaimes et al., 2017; Wadgymar, 2017; Enciso y García, 2014).

Actualmente las universidades apoyan a los alumnos con iniciativas emprendedoras e innovadoras, brindándoles un espacio de interacción con empresarios, emprendedores y directivos, para el desarrollo de nuevos productos, servicios y modelos de negocios que generen valor en las organizaciones y crecimiento económico. Los observatorios de emprendimiento son una herramienta estratégica para el monitoreo y evaluación de los sectores productivos e impactan en la cultura empresarial de los aprendices, incrementando sus potencialidades e incursionando con nuevas competencias (Perdomo et al., 2017; Pérez, 2017).

Los convenios para intercambio académico de los estudiantes y profesores contribuyen al crecimiento personal, profesional y de emprendimiento. Un ejemplo de lo anterior es la experiencia, por un lado, de la crisis económica ecuatoriana $\mathrm{y}$, por otro, la relación de intercambio cultural de la Universidad Autónoma de Yucatán (UAY) y el Instituto Tecnológico de Costa Rica (ITCR). En el caso del cambio del modelo productivo de Ecuador, el sistema universitario y las características del emprendimiento fueron importantes como elementos generadores de riqueza y empleo. En el caso de la UAY y el ITCR, el intercambio cultural y de ideas innovadoras incrementó la seguridad y confianza individual de los estudiantes y profesores (Chaves et al., 2017; López et al., 2017).

Se ha observado que para emprender no basta con entender los principios y prácticas en los negocios y competencias adquiridas en la universidad; sino que la educación es el elemento que complementa al emprendimiento y ayuda a que el individuo observe su conducta, a través de mecanismos sociales y cognitivos. El emprendimiento, además, debe contar con políticas de desarrollo y de responsabilidad social. En Colombia se tiene un caso de éxito, en el que el emprendimiento social posibilitó el desarrollo económico y humano, la innovación social y nuevos modelos de negocios, en las esferas públicas y privadas. En Asia se identificaron elementos en los perfiles de los emprendedores que los convirtieron en empresarios exitosos, siendo estos: habilidades, actitudes, valores, nivel educativo y cultural (Reinoso et al., 2017; Chica et al., 2017; Durán et al., 2017; Hernández et al., 2017b). 
Otro elemento importante del emprendimiento es el uso de las Tecnologías de la Información y Comunicación (TIC's) y el Internet. El uso combinado de estas herramientas empodera y transforma a poblaciones en zonas rurales aisladas, impactando en su desarrollo emprendedor, económico y social. Además, las TIC's y el internet incrementan la productividad en los trabajadores y ayudan a los procesos de capacitación con medios digitales en habilidades técnicas y empresariales. Actualmente se capacita con base en tecnologías de e-learning de segunda generación que se adecúan a las capacidades de atención y agenda de trabajo de los colaboradores (Flores y Salazar, 2017; Ramírez y Moloche, 2017).

Las pequeñas y medianas empresas a nivel mundial representan el $98 \%$ de las unidades económicas en cada país, sus ventajas competitivas están estrechamente relacionadas con la innovación como consecuencia de la investigación, permitiendo ofrecer en los mercados productos y servicios diferenciados, que se transforman en crecimiento sostenible en un contexto globalizado y de libre mercado. Actualmente la cooperación y colaboración entre empresas y clientes son los caminos estratégicos, para crear mercados sin competir, arrebatar, desplazar o conquistar. Las micro, pequeñas y medianas empresas (MiPyMEs) en México, requieren ser pensadas y estudiadas desde el enfoque organizacional. El modelo socioeconómico de las pequeñas y medianas empresas, como metodología de investigación intervención, permite su desarrollo y produce conocimiento científico. (Olmedo, 2017; Ríos, 2017; De la Rosa, 2017; Vázquez, 2002).

Además de las Instituciones de Educación Superior, el gobierno mexicano impulsa la creación de las MiPyMEs. Esto se logra impulsando las relaciones entre empresas, universidades y gobiernos (federal y estatal) para la construcción de entornos emprendedores y la incubación de empresas. Asimismo, las empresas familiares y los procesos sucesorios son factores clave. En este último aspecto, se involucran: la preparación del sucesor, la planificación de la sucesión y las relaciones familiares. Se destaca la importancia de establecer, mantener $y$ fomentar las relaciones interpersonales entre los emprendedores que participan en programas de emprendimiento, para la creación y gestión de microempresas (Malásquez, 2017; González et al., 2017b; Imaginário et al., 2017).

Al respecto, el Fondo Nacional de Apoyo para Empresas de Solidaridad (FONAES) es un programa federal que impulsa a las empresas. Bajo este programa, en Tlaxcala se identificó la necesidad de implementar las siguientes estrategias: mejorar la elección de beneficiarios, capacitar previo diagnóstico, adecuar la planeación al Régimen Fiscal y establecer un sistema de indicadores para monitorear sus resultados. Sin embargo, las empresas familiares también presentan aspectos negativos de acuerdo con su contexto y que pueden implicar el cierre, tales como: falta de información en 
investigación de mercados, programas de apoyo gubernamentales y administración (Reyes et al., 2017; Mazoco et al., 2017).

Asimismo, algunos elementos característicos en la operación de las microempresas son: financiamiento y ventaja competitiva. El financiamiento, generalmente se realiza con ahorros propios o familiares y un porcentaje mínimo se realiza con créditos de instituciones financieras. La ventaja competitiva se refiere principalmente a la gestión del conocimiento (Pacheco et al., 2017; Martínez et al., 2017).

Finalmente, algunos de los retos por atender son: 1) la sobrevaloración de los créditos femeninos que realmente incidan en revertir el empobrecimiento; 2) mejoramiento del financiamiento gubernamental y de la banca para ampliar los servicios de financiamiento con base en créditos, fideicomisos y Fondos Nacionales de Desarrollo; 3) diseño de políticas públicas que fomenten y apoyen el financiamiento de las micro y pequeñas empresas; 4) uso de herramientas para medir y dar seguimiento a las operaciones y de un sistema integral contable administrativo (Pinto et al., 2017; Sánchez, 2017; Ramírez y Malásquez, 2017; Cortez et al., 2017).

Con base en la información anterior, el objetivo de la presente investigación es realizar un estudio de percepción de emprendedores universitarios sobre el impacto del programa escolar ESA en sus empresas, en el municipio de Tehuipango, Veracruz.

\section{Metodología}

Se diseñó un Cuestionario para la Medición de la Percepción Centrado en Emprendedores (CMPCE). La metodología aplicada para el estudio fue de tipo cuantitativa transversal con un alcance exploratorio, descriptivo.

El objetivo del CMPCE es determinar si el programa emprendedor social y autoempleo influye en el nivel emprendedor de los estudiantes. Por lo tanto, se formula la siguiente hipótesis:

El programa emprendedor social y autoempleo influye de manera positiva en el nivel emprendedor de los estudiantes.

El CMPCE consta de 9 reactivos correspondientes a las variables Alumnos Emprendedores (AE), Programa de Emprendimiento (PE) y Empresas Incubadas (EI), en las tablas 1, 2 y 3 se muestra su descripción.

Los primeros tres reactivos corresponden a la variable Alumnos Emprendedores, se muestran en la Tabla No. 1 a continuación:

\begin{tabular}{|c|c|}
\hline \multicolumn{2}{|r|}{ ALUMNOS EMPRENDEDORES } \\
\hline AE1 & ¿Se considera usted emprendedor? \\
\hline AE2 & ¿Es importante para usted ser emprendedor? \\
\hline AE3 & $\begin{array}{l}\text { ¿Las empresas de los emprendedores influyen socioeconómicamente en su } \\
\text { municipio? }\end{array}$ \\
\hline
\end{tabular}


En la Tabla No. 2 ubicada a continuación se muestran los tres reactivos correspondientes a la variable Programa Emprendedor (ítems 4 al 6).

\begin{tabular}{|c|c|}
\hline \multicolumn{2}{|r|}{ PROGRAMA EMPRENDEDOR } \\
\hline PE4 & ¿Considera usted importante el programa emprendedor social y autoempleo? \\
\hline PE5 & $\begin{array}{c}\text { ¿El programa emprendedor social y autoempleo, influyó en su nivel de } \\
\text { emprendimiento? }\end{array}$ \\
\hline PE6 & $\begin{array}{c}\text { ¿Considera usted que el programa emprendedor social y autoempleo, impacta } \\
\text { socioeconómicamente a su municipio? }\end{array}$ \\
\hline
\end{tabular}

Los tres reactivos de la variable Empresas Incubadas (7 al 9) se muestran en la Tabla No. 3.

\begin{tabular}{|c|c|}
\hline \multicolumn{2}{|c|}{ Tabla No. 3. Ítems de Medición de Percepción de Empresas Incubadas en el CMPCE. } \\
\hline \multicolumn{2}{|c|}{ EMPRESAS INCUBADAS } \\
\hline EI7 & ¿Fue importante para usted, desarrollar una microempresa? \\
\hline EI8 & ¿Se encuentra en funcionamiento su microempresa? \\
\hline EI9 & ¿Su microempresa tuvo algún impacto socioeconómico, en su municipio? \\
\hline
\end{tabular}

Estos reactivos se miden en una Escala de Likert de 1 a 5, como se muestra en la siguiente tabla:

\begin{tabular}{|c|c|c|c|c|}
\hline \multicolumn{5}{|c|}{ Tabla 4. Valores de Likert del CMPCE. } \\
\hline 1 & 2 & 3 & 4 & 5 \\
\hline $\begin{array}{c}\text { Totalmente en } \\
\text { desacuerdo }\end{array}$ & En desacuerdo & Indiferente & De acuerdo & $\begin{array}{c}\text { Totalmente de } \\
\text { acuerdo }\end{array}$ \\
\hline
\end{tabular}

La recolección de datos se realizó a una población de 32 estudiantes egresados de las Ingenierías en: Sistemas Computacionales, Desarrollo Comunitario y Gestión Empresarial, mediante el Programa Emprendedor Social y Autoempleo que oferta el Instituto Tecnológico Superior de Zongolica. Al final, se obtuvieron 32 encuestas respondidas. El estudio se realizó en el municipio de Tehuipango, Veracruz, México, en un periodo establecido entre agosto y noviembre de 2017.

El procedimiento diseñado fue el siguiente:

1. Diseño, aplicación y validación de cuestionario con prueba piloto.

2. Una vez validado el cuestionario piloto, se procedió a aplicar el cuestionario final a toda la población de egresados del programa emprendedor social y autoempleo.

La validación se llevó a cabo con la prueba de Chi cuadrada, comparando el nivel de sensibilidad con las variables y factores determinados (Tablas 5, 6 y 7). 


\section{Resultados y discusión}

Los resultados de la encuesta aplicada a 32 ingenieros egresados del programa emprendedor social y autoempleo en los periodos 2015-2016, fueron los siguientes (Tabla 5 y Figura 1):

AE1. ¿Se considera usted emprendedor? 22 Ingenieros respondieron la opción 4 y 8 Ingenieros la opción 5, lo que significa que están de acuerdo y totalmente de acuerdo con ser emprendedores.

AE2. ¿Es importante para usted ser emprendedor? 14 Ingenieros respondieron la opción 4 y 16 Ingenieros la opción 5, lo que indica que es importante para ellos ser emprendedores.

AE3. ¿Las empresas de los emprendedores influyen socioeconómicamente en su municipio? 22 Ingenieros respondieron la opción 4 y 5 Ingenieros la opción 5, lo que significa que están de acuerdo y totalmente de acuerdo con haber influido socioeconómicamente en el municipio.

PE4. ¿Considera usted importante el programa emprendedor social y autoempleo? 15 Ingenieros respondieron la opción 4 y 15 Ingenieros la opción 5, lo que indica que fue importante para ellos haber cursado el programa emprendedor social y autoempleo.

PE5. ¿El programa emprendedor social y autoempleo, influyó en su nivel de emprendimiento? 23 Ingenieros respondieron la opción 4 y 8 Ingenieros la opción 5, lo que significa que el programa emprendedor social y autoempleo si tuvo una influencia en su nivel de emprendimiento.

PE6. ¿Considera usted que el programa emprendedor social y autoempleo, impacta socioeconómicamente a su municipio? 21 Ingenieros respondieron la opción 4 y 8 Ingenieros la opción 5 , lo que indica que el programa emprendedor social y autoempleo si impactó socioeconómicamente en su municipio.

EI7. ¿Fue importante para usted, desarrollar una microempresa? 14 Ingenieros respondieron la opción 4 y 16 Ingenieros la opción 5, lo que significa que fue importante para ellos el haber creado una microempresa.

EI8. ¿Se encuentra en funcionamiento su microempresa? 9 Ingenieros respondieron la opción 4 y 13 Ingenieros la opción 5, lo que indica que más de la mitad de sus empresas se encuentran en funcionamiento.

EI9. ¿Su microempresa tuvo algún impacto socioeconómico, en su municipio? 18 Ingenieros respondieron la opción 4 y 10 Ingenieros la opción 5 , lo que significa que sus microempresas sí tienen un impacto socioeconómico en el municipio. 


\begin{tabular}{|c|c|c|c|c|c|c|c|c|c|}
\hline \multicolumn{10}{|c|}{ Tabla 5. Frecuencias. } \\
\hline No & AE 1 & AE 2 & AE 3 & PE 4 & PE 5 & PE 6 & EI 7 & EI 8 & EI 9 \\
\hline 1 & 0 & 0 & 1 & 0 & 0 & 0 & 0 & 3 & 0 \\
\hline 2 & 1 & 1 & 2 & 0 & 1 & 0 & 1 & 5 & 2 \\
\hline 3 & 1 & 1 & 2 & 2 & 0 & 3 & 1 & 2 & 2 \\
\hline 4 & 22 & 14 & 22 & 15 & 23 & 21 & 14 & 9 & 18 \\
\hline 5 & 8 & 16 & 5 & 15 & 8 & 8 & 16 & 13 & 10 \\
\hline
\end{tabular}

Nivel de Percepción

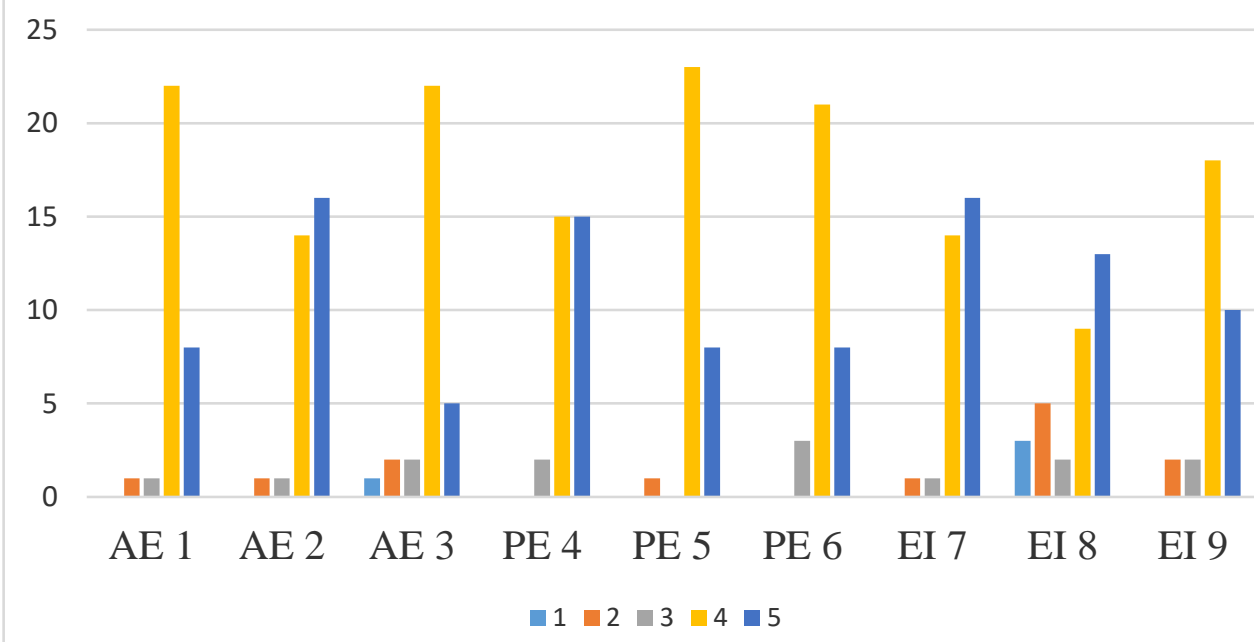

Figura 1. Resultados de la encuesta, con base en la escala Likert.

\begin{tabular}{|c|c|c|c|c|c|c|}
\hline \multicolumn{7}{|c|}{ Tabla 6. Chi cuadrada Nivel de apreciación (Likert) vs. Items. } \\
\hline & Nivel 1 & Nivel 2 & Nivel 3 & Nivel 4 & Nivel 5 & Totales \\
\hline AE 1 & 0 & 1 & 1 & 22 & 8 & 32 \\
\hline AE 2 & 0 & 1 & 1 & 14 & 16 & 32 \\
\hline AE 3 & 1 & 2 & 2 & 22 & 5 & 32 \\
\hline PE 4 & 0 & 0 & 2 & 15 & 15 & 32 \\
\hline PE 5 & 0 & 1 & 0 & 23 & 8 & 32 \\
\hline PE 6 & 0 & 0 & 3 & 21 & 8 & 32 \\
\hline EI 7 & 0 & 1 & 1 & 14 & 16 & 32 \\
\hline EI 8 & 3 & 5 & 2 & 9 & 13 & 32 \\
\hline EI 9 & 0 & 2 & 2 & 18 & 10 & 32 \\
\hline Totales & 4 & 13 & 14 & 158 & 99 & 288 \\
\hline
\end{tabular}




\begin{tabular}{|c|c|c|c|c|c|c|}
\hline \multicolumn{7}{|c|}{ Tabla 7. Estadísticos, Chi calculada. } \\
\hline & Nivel 1 & Nivel 2 & Nivel 3 & Nivel 4 & Nivel 5 & Totales \\
\hline AE 1 & 0.44 & 0.14 & 0.20 & 1.13 & 0.82 & 2.72 \\
\hline AE 2 & 0.44 & 0.14 & 0.20 & 0.72 & 2.27 & 3.77 \\
\hline AE 3 & 0.69 & 0.21 & 0.13 & 1.13 & 3.27 & 5.43 \\
\hline PE 4 & 0.44 & 1.44 & 0.13 & 0.37 & 1.45 & 3.84 \\
\hline PE 5 & 0.44 & 0.14 & 1.56 & 1.69 & 0.82 & 4.64 \\
\hline PE 6 & 0.44 & 1.44 & 1.34 & 0.68 & 0.82 & 4.72 \\
\hline EI 7 & 0.44 & 0.14 & 0.20 & 0.72 & 2.27 & 3.77 \\
\hline EI 8 & 14.69 & 8.75 & 0.13 & 4.17 & 0.36 & 28.11 \\
\hline EI 9 & 0.44 & 0.21 & 0.13 & 0.01 & 0.09 & 0.89 \\
\hline Totales & 18.5 & 12.615385 & 4 & 10.607595 & 12.181818 & $\mathbf{5 7 . 9 0 4 7 9 8}$ \\
\hline \multicolumn{7}{|c|}{} \\
\hline \multicolumn{7}{|l|}{ La Chi calculada es mayor que la Chi teórica, por lo tanto: las variables son dependientes del } \\
\multicolumn{7}{|l|}{ nivel de apreciación. } \\
\hline
\end{tabular}

\section{Conclusión}

De acuerdo con la prueba de Chi cuadrada se comprobó la hipótesis, concluyendo que el programa de emprendimiento sí influyó directamente en el nivel emprendedor de los encuestados, lo que indica que las variables $\mathrm{AE}$, PE y EI son dependientes del nivel de apreciación.

Con base en la tabla 5 de frecuencias, se identifican con mayor incidencia todos los Items excepto el EI8. Lo que significa que el $93.75 \%$ de los encuestados se consideran emprendedores, creen que es importante serlo, consideran importante haber cursado el programa de emprendimiento y mencionan que fue importante haber creado microempresas.

Además, el $90.63 \%$ de la población considera que el programa de emprendimiento impacta directamente en su municipio, el $87.5 \%$ percibe que las empresas tuvieron un impacto socioeconómico en su municipio.

Asimismo, el $84.38 \%$ de los emprendedores identifican que sus empresas influyen socioeconómicamente en su municipio.

Finalmente, el $68.75 \%$ de los emprendedores tienen en funcionamiento sus empresas. Por lo tanto, se propone crear un programa de seguimiento a empresas, que permita fomentar su permanencia en el mercado y elevar el crecimiento socioeconómico del municipio.

\section{References:}

1. Castillo, P. V., Venegas, C. B., Leiva, Y. F., Bennett, S. O., Ortiz, E. P., \& de Souza Neto, B. (2016). Una innovación pedagógica para la formación de universitarios emprendedores. Revista da FAE, 11(2).

2. Chaves-Abarca, R., Cantón-Castillo, L. C. G., Luit-Gonzále, M. D. C., \& García-Lira, A. (2017). Desarrollo de emprendedores entre la 
Universidad Autónoma de Yucatán y el Instituto Tecnológico de Costa Rica. Revista Tecnología en Marcha, 30(2), 128-141.

3. Chica, M. F., Posso, M. I., \& Montoya, J. C. (2017). Importancia del emprendimiento social en Colombia. Documentos de Trabajo ECACEN, (2).

4. Cortez, J. H. Z., Guajardo, J. E. G., González, L. G., \& Martínez, M. M. E. B. (2017). Propuesta de una herramienta de apoyo para la información en la toma de decisiones en pequeñas y medianas empresas de la región centro del Estado de Coahuila. Revista de Investigación en Ciencias y Administración, 9(16), 5-26.

5. De la Rosa Alburquerque, A. (2017). Hacia la emergencia de un nuevo objeto de estudio: la micro, pequeña y mediana organización. Iztapalapa, (56), 129-174.

6. Duran, S., Fuenmayor, A., Cárdenas, S., \& Hernández, R. (2017). Emprendimiento como proceso de responsabilidad social en instituciones de educación superior en Colombia y Venezuela. Desarrollo gerencial, 8(2).

7. Enciso, J. A. G., \& García, R. A. C. Vinculación universitaria con el ámbito productivo: la gestión institucional de incubadoras en la Universidad Autónoma del Estado de México.

8. Flores, S. G. T., \& Salazar, R. L. C. (2017). Educación Continua mediada en Redes sociales como estrategia para el Emprendimiento e Innovación Social en Zonas Rurales. Memorias del Encuentro Internacional de Educación a Distancia, 5(5).

9. Gonzalez-Garcia, G., Estrada-Gutierrez, C. E., Leite, E., \& AlvarezBotello, J. (2017). Aportaciones al diseño de formación de emprendedores desde la medición de actitudes del estudiante universitario. CASO UAEMEX-UPTC (2016). HOLOS, 2, 290-300.

10. González, M. A. M., Benítez, H. A. U., \& Tamayo, E. J. G. (2017). Factores determinantes del éxito del proceso sucesorio en empresas familiares de Dzityá, Yucatán. Red Internacional de Investigadores en Competitividad, 4(1).

11. Hernández, F. O., Moctezuma, V. O., \& Martinelli, J. M. (2017). La nueva gestión del conocimiento y el desarrollo del modelo cultural en los jóvenes universitarios. Red Internacional de Investigadores en Competitividad, 2(1).

12. Hernández, Z. T., López, L. R. C., \& Galaviz, J. L. F. (2017). Análisis de habilidades gerenciales de empresarios asiáticos, que empezaron como microempresas y lograron crecer a empresas de clase mundial. Revista de Investigación en Ciencias y Administración, 6(10), 369-386. 
13. Imaginário, S., Cristo, E., de Jesus, S. N., \& Morais, F. (2017). Creación y gestión de microempresas en el salón de clase: opiniones de estudiantes y profesores participantes en el Programa Emprender en la Escuela. Avances en Psicología Latinoamericana, 35(1), 42.

14. Jaimes, E. I. G., Silva, M. G. M., \& Zumpango, C. U. U. (2017). Egresados universitarios y su pertinencia para el ingreso a la empleabilidad empresarial o la auto-empleabilidad. Revista Electrónica de Psicología Iztacala, 20(1), 168.

15. Krauss, C. (2011). Actitudes emprendedoras de los estudiantes universitarios: El caso de la Universidad Católica del Uruguay.

16. López, E. G. M., Carreño, O. F. M., Alarcón, L. F. J., \& Reinoso, M. V. A. (2017). El emprendimiento en el sistema universitario. Revista Didasc@ lia: Didáctica y Educación. ISSN 2224-2643, 8(1), 163178.

17. Malásquez, P. M. C. (2017). Incubadoras de empresas en México en el contexto de las relaciones universidad, empresa, gobierno. Revista de Investigación en Ciencias y Administración, 6(11), 87-117.

18. Maldonado, E. F. N., Chugcho, C. B. S., Valarezo, J. K. O., \& Ramón, D. I. R. (2017). Análisis de Actitud Emprendedora en Estudiantes Universitarios: una Perspectiva de Género. INNOVA Research Journal, 2(8), 56-63.

19. Martínez, R. M., Chávez, R. F. R., \& Taylor, V. A. (2017). La gestión del conocimiento en las microempresas familiares formales de servicios en Uruapan, Michoacan. Revista de Investigación en Ciencias y Administración, 6(11), 451-469.

20. Mazoco, E. M. R., Carmona, E. A., \& Santiago, L. P. B. (2017). Analysis Of Closing Conditions Of Smes In Zacatecas-Guadalupe In Mexico, Analisis De Condiciones De Cierre De Mipymes Comerciales En La Zona Conurbada Zacatecas-Guadalupe En Mexico. Revista Global de Negocios, 5(7), 45-54.

21. Millán, F. J., Jaramillo, M. J., \& Chávez, M. A. P. (2017). Factores que inciden en la intención emprendedora de estudiantes del Centro Universitario Temascaltepec. Revista Venezolana de Gerencia, 22(78), 210-231.

22. Montiel, O., \& Lucio, C. I. R. (2017). ¿Lo volvería a hacer? Revisitando la implementación del paradigma del emprendimiento en una universidad de México. Retos, 7(14), 147-165.

23. Moriano León, J. A., Palaci Descals, F. J., \& Morales Domínguez, J. F. (2006). El perfil psicosocial del emprendedor universitario. Revista de Psicología del Trabajo y de las Organizaciones, 22(1).

24. Olmedo, W. H. N. (2017). Investigación e innovación, factores de crecimiento en las PYMES. Revista Publicando, 4(12 (2)), 254-268. 
25. Olmos, R. E., \& Castillo, M. Á. S. (2007). La actitud emprendedora durante la vida académica de los estudiantes universitarios. Cuadernos de estudios empresariales, 17, 95-116.

26. Pacheco, N. P. G., Pinto, P. S., \& Pacheco, N. J. G. (2017). Estudio de impacto del financiamiento microempresarial en Tunja (Colombia) y Arequipa (Perú). Hacia una cultura de éxito financiero empresarial. TZHOECOEN, 9(2).

27. Perdomo, P. E. Á., Alcívar, M. A. A., Salvatierra, J. X. B., Martínez, M. E. R., \& Vilcacundo, J. L. G. (2017). La innovación y el emprendimiento: necesidades en la educación superior. Revista Didasc@ lia: Didáctica y Educación. ISSN 2224-2643, 7(4), 229246.

28. Pérez, J. (2017). Una propuesta de observatorios de emprendimiento para el Centro de Servicios Financieros del Servicio Nacional de Aprendizaje. Revista Finnova: Investigacion e Innovacion Financiera y Organizacional, 2(3), 29-43.

29. Pinto, E. D. C. A., Pablos, E. T., \& Barragán, F. M. (2017). Microcrédito y pobreza. La experiencia del programa Microempresas Sociales de Banmujer en Chiapas. Economía Sociedad y Territorio, (1), 809-835.

30. Ramírez, A. R. R., \& Malásquez, P. M. C. (2017). Problemática y alternativas de financiamiento para las micros y pequeñas empresas familiares de michoacán: estudio de caso. Revista de Investigación en Ciencias y Administración, 8(15), 127-168.

31. Ramírez, M. J., \& Moloche, G. (2017). Costos de Información en Programas de Formación de Habilidades en Pymes: Un Experimento de Campo en Servicios Publicitarios. Revista Cientifica TECNIA, 26(2), 88 .

32. Reinoso, M. V. A., Alarcón, L. F. J., \& López, E. G. M. (2017). Emprendimiento corporativo para las empresas. Una visión desde la universidad contemporánea. Revista Didasc@ lia: Didáctica y Educación. ISSN 2224-2643, 8(1).

33. Reyes, A. B., Lara, M. A. C., \& Sánchez, C. U. S. (2017). Impacto generado en las microempresas beneficiadas por el apoyo formación empresarial del FONAES en el estado de Tlaxcala. Red Internacional de Investigadores en Competitividad, 3(1).

34. Rios, C. B. (2017). La cooperación y la autopoiesis, como estrategia de cambio al mercado: caso microempresas de trasformación en el estado de Veracruz, México. Ciencia Administrativa, (2), 143-153.

35. Sánchez, J. I. J. (2017). Nuevas modalidades de financiación para microempresas. Puente, 8(2), 61-72. 
36. Vázquez, G. M. El modelo socioeconómico como metodología de vinculación universidad-empresa.

37. Wadgymar, K. M. (2017). La apuesta de México al emprendimiento social: Hult Prize, el "Premio Nobel de los estudiantes". MUUCH'XÍIMBAL CAMINEMOS JUNTOS, (4). 